\title{
A Study on Space Fitting between the Spatial Links of Services and Economic Openness in Guangdong Province
}

\author{
HUANG Dawei ${ }^{1,2, a}$ \\ (1. School of Management, Shenzhen Polytechnic, Shenzhen 518055, China, \\ 2. School of Urban Planning and management, Harbin Institute of Technology Shenzhen Graduate \\ School, Shenzhen 518055, China) \\ adaweihuang@163.com
}

Keyword: Spatial gravity model; Spatial links of Services; Services agglomeration; Economic openness; Spatial fitting

\begin{abstract}
Spatial gravity model has been used and indicators, namely, potential development capacity and acceleration, agglomeration force and acceleration, ability of receiving radiation and traction acceleration of Service Industry have been created, based on the Services' data of 21 prefecture-level city from year 2001, 2005, 2008, 2012 in Guangdong province, to discover municipal Services’ agglomeration level, "quality”, spatial links and space-time evolution process of spatial framework. Municipal Services' spatial connection diagrams have been made based on the regional strength of the interaction, and with comparison a significant coupling relationship has been proved among potential development capacity, acceleration and spatial links, and the tightness of spatial connection of regional service industry does play a significant role in promoting the space-time evolution of Services' spatial structure, which means with Services' spatial links turning gradually closer, the spatial structure of Services displays more complexity, transforming from original single-core structure to multi-core structure, and lastly being stable at complex network structure, regional services integration framework begins to emerge. Moreover, according to data of spatial links of Services, agglomeration level and level of economic openness from the four time section, a corresponding spatial distribution figure has been drawn by R-language to explore their relationship in spatial fitting. It shows that they are perfectly fitting in spatial distribution, and all of them concentrate their distribution in the Pearl River Delta region, which indicates that economic openness is the one significant driving factor enhancing the spatial links of Services and agglomeration level, and agglomeration level improving and lingks going closer always make contributions to an accelerated trans-regional and cross-border circulation of population, information, capital and other resources, which indirectly enhance regional economic openness.
\end{abstract}

In recent years, with the global economic service, the academic community on the spatial distribution of the service industry has gradually increased [1], a large number of theoretical and empirical research focused on the proliferation of productive services industry space [2,3], spatial heterogeneity distribution [4], Industrial spatial structure and evolution analysis [5,6] and other directions, the results are quite abundant. With the country into the new economic normal, eight vertical and eight horizontal space planning makes the economic space is becoming increasingly close, the previous attention to the economic spatial distribution of academic research will gradually shift to economic space, economic network structure. In addition, Guangdong Province, as the pioneer province of China's economic opening up, the development of its regional service industry and the spatial connection must have a significant relationship with the degree of economic openness. In the past, there are few researches on the spatial and spatial Economic openness of the spatial distribution of the relationship between the empirical studies to explore the intrinsic relationship between the two.

To sum up, this study will first analyze the spatial connection of service space and the spatial network structure from the perspective of spatial interaction. On this basis, further analysis of the service industry spatial contact, network structure and economic openness of the spatial fitting match to examine the internal relationship between service industry and economic openness. 


\section{The basis of service space contact}

The service industry spatial statistical analysis based on the data of 2001, 2005, 2008, 2012 of the four years of 21 cities in Guangdong Province, the data are derived from the corresponding year "statistical yearbook of Guangdong province", "Chinese City Statistical Yearbook".

\subsection{The Spatial Gravity Model of service industry}

In recent years, the physics of the law of gravitation is widely used in the study of geography and regional economic subjects, scholars through certain variables set, construct a gravity model [7], according to geographical distance attenuation, spatial interaction issues such as research $[8,9]$. On the basis of previous studies [10-12], this study constructs the Spatial Gravity Model of service industry as follows:

$$
F_{i j}=\frac{k Q_{i} Q_{j}}{R_{i j}^{2}}
$$

Where: $F_{i j}$ is the spatial interaction between services of regional $i$ and regional $j$; $k$ is the gravitational constant, the default is $1 ; Q_{i}, Q j$ are the "quality" of the service industry in the region $i, j ; R_{i j}^{2}$ is the regioni and the region $j$, where the commute distance is taken as a measure of the distance.

The "quality" of the development of the service industry in the region $i$ can be measured by the geometric mean of the value of the regional service industry and the total number of employees in the service sector (as shown in equation (2)):

$$
Q_{i}=\sqrt{G D P_{i} \times P O P_{i}}
$$

It can be seen from the formula that the inter-regional interaction force $F_{i j}$ reflects the inter-regional service industry interrelated, the strength of interaction, $F_{i j}$ is proportional to the level of regional service industry development, inversely proportional to the distance between regions. From the perspective of regional economic agglomeration, $F_{i j}$ can reflect the radiation intensity of the service area (developed area) for the surrounding areas (underdeveloped areas), but also reflect the reception capacity of the surrounding areas for the service area.

In addition, in order to more clearly describe the spatial connection characteristics of regional service industry, this study also introduces the spatial agglomeration characteristic variables and regional service industry development potential variables to enrich the gravitational model.Location business is the regional economy in the portraying of industrial agglomeration, measure the specialized departments of the common indicators,therefore, by calculating the service industry value location quotient $L Q_{i}$ to describe the spatial clustering characteristics of regional service industry, The size of the location value $L Q_{i}$ represents the degree of specialization and agglomeration of the service industry in the region,more precisely, the region for the service industry elements of the ability to attract capacity, which is an important factor between the regional elements of the flow of indicators, will inevitably affect the spatial structure of the service industry. $L Q_{i}$ formula as shown in (3), where $G D P_{i s}, G D P_{i} 、 G D P_{s} 、 G D P$, respectively, on behalf of the region $i$ total output value of services, GDP of regional $i$, total output value of Guangdong Province, Guangdong Province, GDP.

$$
L Q_{i}=\frac{G D P_{i s} / G D P_{i}}{G D P_{s} / G D P}
$$

On this basis, this study draws on the construction of financial potential in Yang Zhimin, HuaXiangyu, Ye Yafen et al. (2015) [11], and adds a weight ( $\left.L Q_{i}-L Q_{j}\right)$, that is, the difference in the degree of specialization between regions to modify the role of regional service industry gravitation, innovative construction of a regional service industry development potential variable $F_{D P}$, used to describe the space in the relationship between the characteristics of the regional service industry with the development potential, such a (4) and (5), as shown in the formulas (4) and (5): (2) 


$$
A=\sum_{j}\left(L Q_{i}-L Q_{j}\right) * F_{i j}
$$

Where: $F_{D P i}$ is the development potential of region $i, A$ is the concentration of radiation, $L Q_{i}$, $F_{i j}$ is the spatial interaction between the service area of the region $i$ and the regional service area $i$ and region $j$. It is necessary to pay attention to the positive and negative of $A$, when $A$ is positive, the concentration of $i$ is greater than the received radiation intensity, that is, the development potential depends mainly on its own gathering capacity; when $A$ is negative, the concentration of agglomeration is less than the received radiation intensity, and the development potential mainly depends on its own ability to receive radiation in the developed areas. $A=0$, it means that the concentration of $i$ is equivalent to the radiation intensity. That is to say, the development potential of the regionimainly come from two aspects, namely, the ability of its own gathering and the ability to accept radiation in developed areas. Through the above analysis, this study can also find the capacity of the city-level city service industry and the ability to accept the radiation of the service area in the surrounding areas, as shown in the formulas (6) and (7).

$$
\begin{aligned}
F_{\text {agglo }} i & =\sum_{j}\left(L Q_{i}-L Q_{j}\right) * F_{i j}, L Q_{i}-L Q_{j} \geq 0 \\
& F_{\text {rad } i}=\sum_{j}\left(L Q_{i}-L Q_{j}\right) * F_{i j}, L Q_{i}-L Q_{j}<0
\end{aligned}
$$

Through the above analysis, we can get the "quality" $Q_{i}$ of service industries in prefecture level cities,latent capacity of service industry development $F_{D P i}$, concentration capacity of service industry $F_{\text {agglo }}$, and acceptance of services in the surrounding areasradiation capacity $F_{\text {rad }}$ these four indicators. According to the new law of Newton's second law, that is, $F=m a$ can be calculated out of the region's own service industry, "quality" factors in the region of the potential development of service potential $a_{d p i}$, agglomeration acceleration $a_{\text {agglo }}$, radiation traction acceleration $a_{\text {rad }}$, which can be estimated The main driving force of the spatial structure change of the service area in the region and the driving acceleration.

\subsection{Spatial clustering of service industry and analysis of spatial gravity}

\subsubsection{Gravity analysis of service industry}

The development of regional service industry is not only affected by the degree of specialization of the service sector and the "quality" of the service industry in the region, but also has a close relationship with the spatial interaction of the service industry, manifesting itself as a gathering force and receiving the surrounding area Radiation force. Therefore, according to the formulas (4), (5), (6), (7) and Newton's second law F=ma,respectively, the calculation of 2001, 2005, 2008, 2012 four years in Guangdong Province 21 prefecture-level city service industry development potential $F_{D P i}$, service industry gathering capacity $F_{\text {agglo }}$ and the surrounding areas to receive service capacity radiation $F_{\text {rad }}$, service industry potential development accelerated $a_{d p i}$, agglomeration acceleration $a_{\text {agglo }}$, radiation traction acceleration $a_{\text {rad }}$, through these mechanical indicators to describe the inter- Service industry interaction and spatial layout of the development, as shown in Table 1 for the development of services in Guangdong Province, the three mechanical indicators data. First of all, look at the development potential of the service industry $F_{D P i}$ changes in time and space, it is not difficult to find the potential value of Guangzhou and Foshan far ahead of other regions, but also in the timeline there is a stable advantage, followed by Shenzhen, Dongguan, Jiangmen and Zhongshan four cities have a certain potential advantage. From the perspective of the two sources of potential, the spatial and temporal distribution of the cohesive force shows a significant difference with the temporal and spatial distribution of the potential, mainly in Shenzhen and Shantou. And the distribution of radiation capacity and the distribution of latent capacity is basically consistent, the difference between the points in Guangzhou, Shenzhen, the two cities; 
Guangzhou is in the core of the gathering, no city of Guangzhou radiation effects, and thus radiation acceptance is unknown, the default is zero; Shenzhen is also in the core position of the gathering, the level is second only to Guangzhou, so the potential is mainly from the gathering capacity, radiation acceptance is weak; in addition to the other cities outside the basic performance potential is mainly derived from the development of radiation capacity feature.

Tab. 1 Potential development capacity, agglomeration force and ability of receiving radiation of Service Industry (unit: hundred million person yuan / sq km)

\begin{tabular}{cccccccccccccc}
\hline \multirow{2}{*}{ City } & \multicolumn{3}{c}{2001} & \multicolumn{3}{c}{2005} & \multicolumn{3}{c}{2008} & \multicolumn{4}{c}{2012} \\
\cline { 2 - 7 } & $F_{d p}$ & $F_{\text {aqglo }}$ & $F_{\text {rad }}$ & $F_{d p}$ & $F_{\text {aqqlo }}$ & $F_{\text {rad }}$ & $F_{d p}$ & $F_{\text {agqlo }}$ & $F_{\text {rad }}$ & $F_{\text {dp }}$ & $F_{\text {aqglo }}$ & $F_{\text {rad }}$ \\
\hline Guangzhou & 37.86 & 37.86 & 0.00 & 133.19 & 133.19 & 0.00 & 272.11 & 272.11 & 0.00 & 586.68 & 586.68 & 0.00 \\
Shaoguan & 0.22 & 0.01 & 0.21 & 0.38 & 0.04 & 0.33 & 0.88 & 0.10 & 0.78 & 1.70 & 0.27 & 1.44 \\
Shenzhen & 3.54 & 1.98 & 1.56 & 13.88 & 7.30 & 6.57 & 31.39 & 22.29 & 9.10 & 72.74 & 50.89 & 21.85 \\
Zhuhai & 0.85 & 0.17 & 0.68 & 2.68 & 0.94 & 1.74 & 7.14 & 1.59 & 5.55 & 15.91 & 2.51 & 13.40 \\
Shantou & 0.78 & 0.73 & 0.05 & 0.86 & 0.70 & 0.16 & 1.44 & 1.04 & 0.41 & 3.12 & 2.18 & 0.94 \\
Foshan & 27.16 & 0.51 & 26.65 & 103.32 & 0.14 & 103.18 & 223.15 & 0.16 & 222.98 & 481.02 & 0.12 & 480.91 \\
Jiangmen & 2.21 & 0.43 & 1.78 & 5.58 & 0.41 & 5.17 & 12.54 & 0.36 & 12.17 & 24.77 & 1.66 & 23.11 \\
Zhanjiang & 0.14 & 0.05 & 0.09 & 0.41 & 0.00 & 0.41 & 0.90 & 0.00 & 0.90 & 1.17 & 0.10 & 1.07 \\
Maoming & 0.21 & 0.01 & 0.20 & 0.47 & 0.14 & 0.33 & 1.02 & 0.46 & 0.56 & 1.68 & 0.31 & 1.37 \\
Zhaoqing & 2.03 & 0.12 & 1.91 & 2.11 & 0.64 & 1.47 & 4.39 & 1.07 & 3.32 & 11.60 & 0.51 & 11.09 \\
Huizhou & 1.67 & 0.00 & 1.67 & 3.60 & 0.00 & 3.60 & 8.04 & 0.11 & 7.93 & 21.49 & 0.12 & 21.37 \\
Meizhou & 0.13 & 0.02 & 0.12 & 0.25 & 0.01 & 0.24 & 0.48 & 0.06 & 0.42 & 1.07 & 0.35 & 0.72 \\
Shanwei & 0.15 & 0.03 & 0.13 & 0.33 & 0.01 & 0.32 & 0.74 & 0.04 & 0.70 & 1.79 & 0.06 & 1.74 \\
Heyuan & 0.19 & 0.03 & 0.16 & 0.46 & 0.08 & 0.37 & 1.39 & 0.01 & 1.38 & 2.87 & 0.02 & 2.85 \\
Yangjiang & 0.20 & 0.00 & 0.20 & 0.39 & 0.01 & 0.38 & 0.80 & 0.06 & 0.74 & 2.15 & 0.01 & 2.15 \\
Qingyuan & 1.23 & 0.00 & 1.23 & 2.28 & 0.09 & 2.19 & 6.86 & 0.00 & 6.86 & 10.19 & 1.09 & 9.11 \\
Dongguan & 2.88 & 0.22 & 2.65 & 11.77 & 1.23 & 10.53 & 21.90 & 5.18 & 16.72 & 42.78 & 10.76 & 32.03 \\
Zhongshan & 1.71 & 0.03 & 1.68 & 6.17 & 0.02 & 6.15 & 12.16 & 0.65 & 11.51 & 28.18 & 1.64 & 26.54 \\
Chaozhou & 0.40 & 0.15 & 0.25 & 0.42 & 0.04 & 0.39 & 0.75 & 0.24 & 0.51 & 1.90 & 0.89 & 1.01 \\
Jieyang & 0.73 & 0.00 & 0.73 & 0.63 & 0.00 & 0.62 & 1.48 & 0.00 & 1.48 & 4.40 & 0.00 & 4.40 \\
Yunfu & 0.39 & 0.00 & 0.39 & 0.87 & 0.00 & 0.87 & 1.53 & 0.00 & 1.53 & 3.12 & 0.00 & 3.12 \\
\hline
\end{tabular}

This result shows that the development of service industry in Guangdong Province to the region itself, the main gathering capacity, radiation acceptance capacity, supplemented by the basic situation. In addition, the development of service industry in Guangdong Province is still in the main gathering place, radiation supplemented by the situation, that is, Guangzhou, Shenzhen, Dongguan and other areas as the main gathering center, and the gathering center is basically established and stable, the influence of the surrounding areas is changing from agglomeration to radiation, and the corresponding service circle structure gradually achieves the change of "nuclear-type" - "band structure" - "network type". From the analysis results, the influence of the concentration of agglomeration center on the surrounding areas in Guangdong Province is gradually weakened, and the radiation effect tends to be enhanced.

\section{The spatial linkage of service industry and the evolution of spatial structure}

This paper divides the size of spatial gravity into five ranges according to the range of actual data, which can be used to map the spatial distribution of service industry in Guangdong Province through the combined effect of regional gravity, including the combination of force and radiation."0.5 billion yuan / square kilometer", "0.5-1 million yuan / square kilometers", "1-2 billion yuan / square kilometers", "2-10 billion yuan / square kilometers", "> 1 billion person / square kilometers "(note that the $<0.5$ line in the figure is white, because the upper limit of this interval is very small, so this part of the hidden connection, the actual existence of the interaction), in this 
foundation On the drawing of 2001,2005,2008,2012 four years in Guangdong Province 21 prefecture-level city service industry space map, as shown in Figure (1) below. In the figure, the lines indicate the interaction between the regions, and the thickness and color of the lines indicate the strength of the interaction.

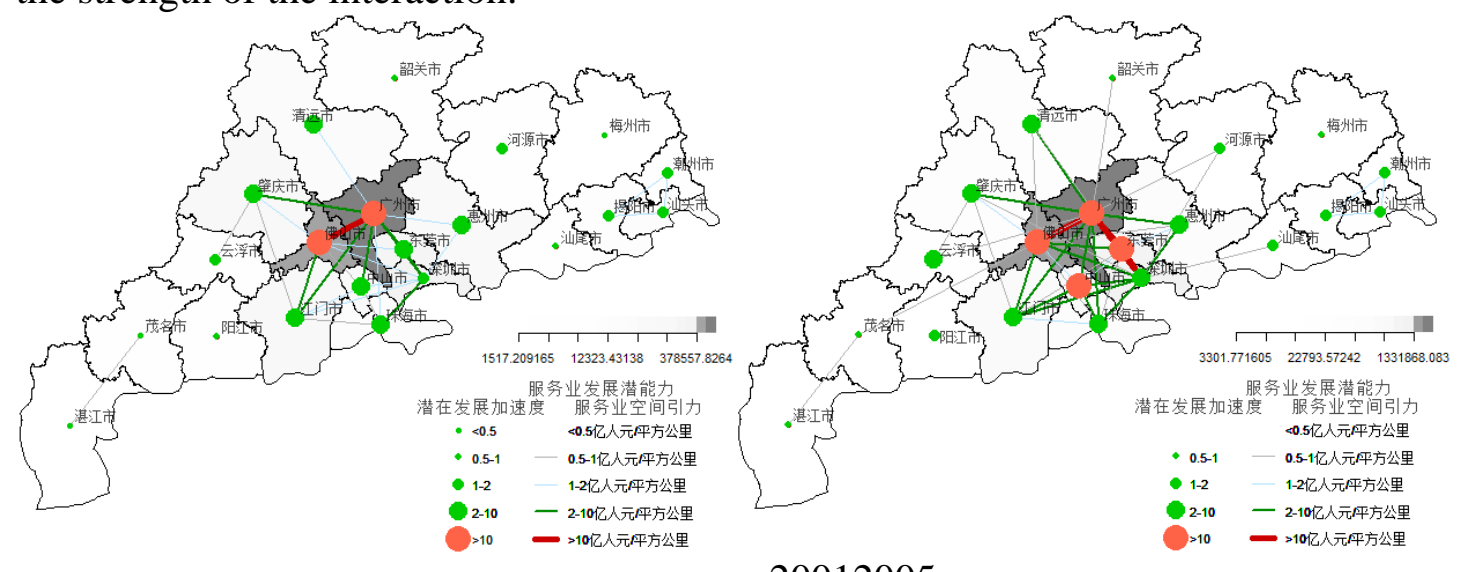

20012005

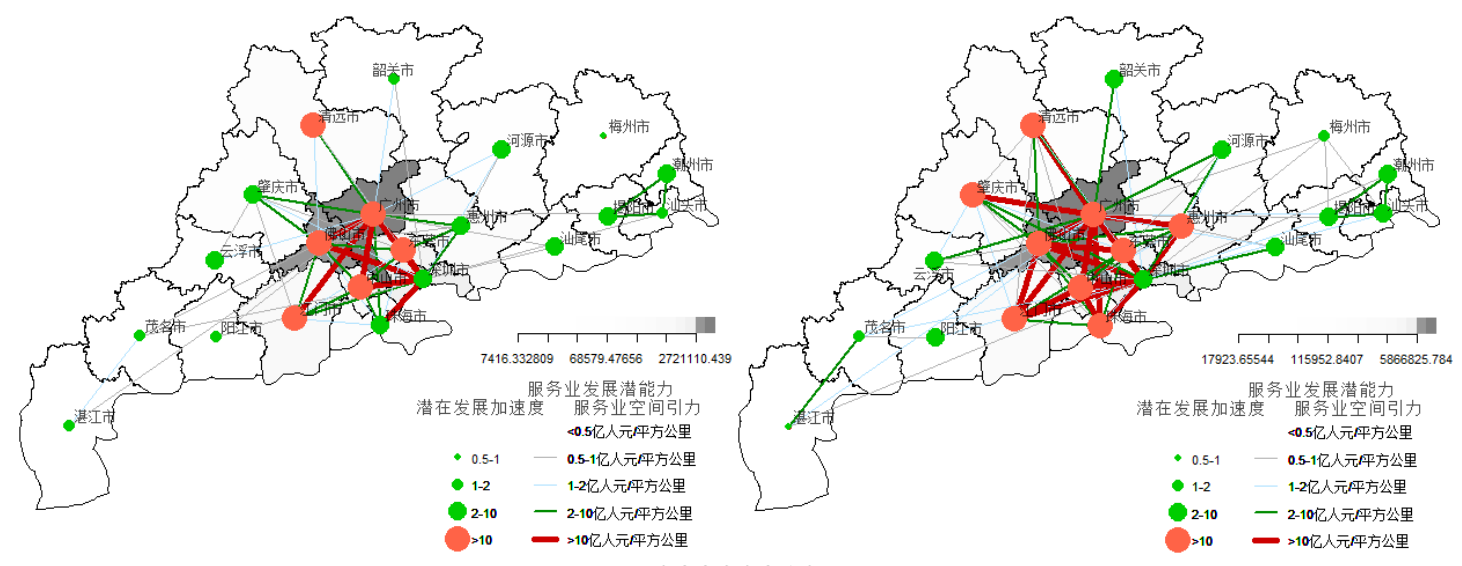

20082012

Fig. 1Spatial links of Services

First of all, from the service industry development potential and potential acceleration point of view to analyze,in terms of latent capacity, the spatial distribution of the potential capacity of the four time sections is basically the same, mainly the development potential of the two cities in Guangzhou and Foshan is the most significant, far more than other cities. In terms of potential acceleration, the four time sections show significant evolutionary characteristics. At the beginning of 2001, the area with the largest acceleration area $(>10)$ was the same as the largest area of potential capacity, that is, only two cities in Guangzhou and Foshan. By 2005, there were two cities in Zhongshan and Dongguan with the largest acceleration area. The addition of Qingyuan and Jiangmen; more than in 2012 Huizhou and Zhuhai; overall, the potential and potential acceleration are increasing year by year. It is noteworthy that Shenzhen does not appear in the potential and potential development of the acceleration of the most favorable areas, which seems to be inconsistent with the facts. This study has been analyzed, the reason why Shenzhen did not appear in the above two indicators because the overall service industry in Shenzhen, "high quality", and Shenzhen service industry potential mainly from the gathering force, through the acceptance of radiation potential Capacity is very weak, much lower than other regions (except Guangzhou, Guangzhou potential all from the concentration), which is leading to the potential of Shenzhen, the main reason for weakness in other regions.

Secondly, from the point of view of spatial connection, the interaction between cities is increasing year by year. At the beginning of the period, the interaction between regions in 2001 was concentrated in the range of "<0.5" and "0.5-1", with less than 10 Green lines (in the"1-2"), 1 Red line (in the " $>=10 "$, Guangzhou - Foshan), the connection points are mainly concentrated in Guangzhou and the surrounding cities, the overall degree of contact is relatively low; in 2005 as a 
whole, the size of gravity is still mainly concentrated in the first two intervals, but compared to a certain degree in 2001 to improve the red line from one to four (Guangzhou - Foshan, Guangzhou Shenzhen, Guangzhou - Dongguan, Shenzhen - Dongguan), the connection point is still mainly concentrated in Guangzhou and the surrounding cities, Shenzhen and Dongguan, the connection density has increased; look again in 2008, Guangdong Province because of its unique geographical relationship by the financial crisis, the impact of the overall connection trend and Similar to the 2005, but the space force has a corresponding increase (green line and red line significantly increased), the connection point is still mainly concentrated in Guangzhou, Shenzhen, Dongguan, the three cities around; to 2012, Guangdong Province, the rapid development of services, As a result of the inter-regional subway, high-speed rail, inter-city and other traffic infrastructure and a substantial increase in the service sector planning in Guangdong Province together, making the regional service industry significantly increased spatial gravity, spatial links also improved significantly, Mainly concentrated in the "1-2,2-10,> = 10" within the range of three, and the green line, the red line increased significantly, the distribution points are concentrated in Guangzhou, Shenzhen, Dongguan, Foshan, Zhuhai and other regions.

Finally, the study of the spatial structure of service industry in Guangdong Province, it is not difficult to see from the figure that the spatial structure of the service industry in Guangdong Province in 2001 mainly shows the "core - edge" structure of Guangzhou, which is a significant "single core"; By 2005, this structure was gradually broken, forming a "multi-core" type "core-edge" structure with Guangzhou, Shenzhen and Dongguan as the gathering point;2008 is basically the same as in 2005, the difference is that the service industry in Zhongshan and Zhuhai agglomeration situation is increasing, and gradually into the "core" circle, the spatial structure from the "core - edge" to "circle structure";By 2012, the spatial structure of the service industry in Guangdong Province evolved from the "circle structure" of 2008 to the "spatial network structure". The spatial layout of the service industry was more complicated, and the regional links were becoming more and more closely. Development of the surrounding areas of the impact of the development of the service industry gradually increased.

\section{The service industry agglomeration, spatial contact and economic open space fit}

During the early period of reform and opening up, the economic development of Guangdong Province mainly relied on the transfer of manufacturing industry in Hong Kong and Taiwan. Through the rise of a large number of foundries, the rapid development of Guangdong's economy with the Pearl River Delta as the engine,to achieve the rapid development of agricultural society in Guangdong Province to the industrial society, the rapid development of non-agricultural economy. After more than 30 years of reform and opening up the policy advantage to guide, coupled with China's new normal industrial structure adjustment and vigorously promote innovation and entrepreneurship, Guangdong Province, Shenzhen, Shenzhen, two core cities to achieve the rise of high-end manufacturing, low-end manufacturing To the surrounding transfer, service industry agglomeration development and spatial links to enhance the economic structure of service change. In the Guangdong Province, the economy has a relatively high degree of openness in the Pearl River Delta region to complete the economic service transformation, and the relatively low degree of economic opening in western Guangdong, northeastern Guangdong is a significant economic backwardness, the slow development of the service industry. Obviously, the level of economic openness directly affects the changes of economic structure in Guangdong Province, the convergence of service industry and the strength of spatial links of service industry. This is in line with previous studies on the positive correlation between economic openness and regional service development. Therefore, this study examines the logical relationship between economic openness and service openness from the perspective of the agglomeration level of the service industry and the spatial connection of the service industry.

In the past studies, the indicators of economic openness are the dependence of foreign trade, the dependence on foreign investment, the amount of foreign trade, the amount of direct investmentand so on, as well as the multi-indicator measures[13,14]. Because this study is a study of the spatial 
fitting relationship between Guangdong Province's economic openness and spatial clustering and spatial connection of service industry, the choice of foreign trade dependence and foreign dependence on the characteristics of economic and social development in Guangdong Province is taken as a measure of economic openness index, that is, according to the regional total import and export volume and the actual use of foreign investment and the proportion of GDP share of the region to represent the level of economic openness, and calculate the 2001,2005,2008,2012 four time section of the Guangdong Province The level of regional openness level data, the regional area of service industry value of the location and business services spatial gravitational mapping spatial distribution map to achieve service industry agglomeration, spatial contact and economic openness distribution of the spatial fitting, as shown in Figure 2.

First, the degree of competition in areas with high economic openness and the agglomeration area of service industry is increasing. The output points of the areas with higher color are generally larger, and the quotient values are basically in the range of the first two ranges, that is in the forefront of the region. Guangdong Province, Foshan, Zhuhai and other regions, the overall trend of change for the Guangzhou, Shenzhen as the core to Dongguan, Zhongshan, Jiangmen, Huizhou and other neighboring cities in the spread of the city. Specifically, in 2001 the service industry gathered in the high degree of economic opening in Guangzhou (the highest is Shenzhen, Zhuhai, Huizhou and Zhongshan four prefecture-level city, the provincial capital Guangzhou followed);In 2005, the overall economic openness of Guangdong Province and the service value of the service industry were raised. The service industry agglomeration was gathered from the single center of Guangzhou in 2001 to the multi-center of Guangzhou, Shenzhen, Zhuhai and Zhaoqing, Zhuhai is the highest degree of economic open areas, Guangzhou, Zhaoqing followed;In 2008, by the international financial crisis, Guangdong Province, foreign economic and trade damage, especially in Dongguan, Huizhou area, but this time the service industry cluster is still high with the economic opening of Shenzhen, Dongguan and Guangzhou coincide.In 2012, the economic crisis sequelae highlighted the international manufacturing industry evacuated from China, leading to the overall level of economic openness in Guangdong Province decreased significantly, when the service industry agglomeration point basically stable in the "Guangzhou - Shenzhen - Dongguan" three central agglomeration situation, and the surrounding area by The radiation of the gathering center, the degree of specialization of the service industry is also gradually increased, the overall distribution and economic openness of the distribution of high and low distribution is more significant.t.

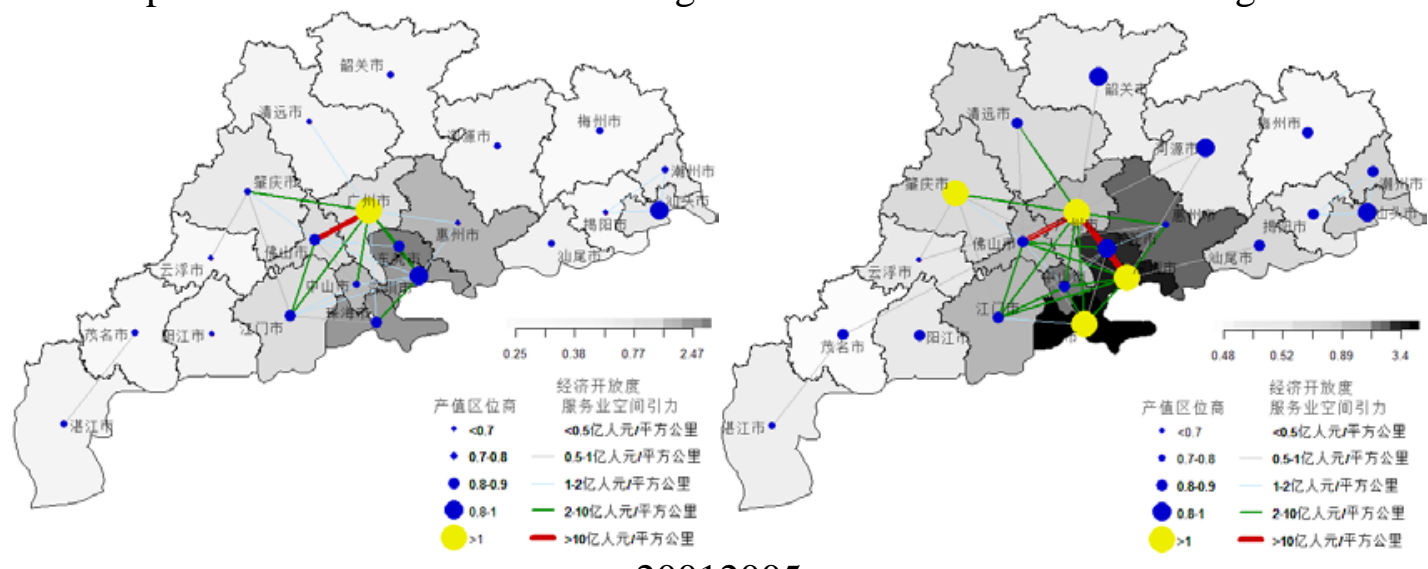

20012005 


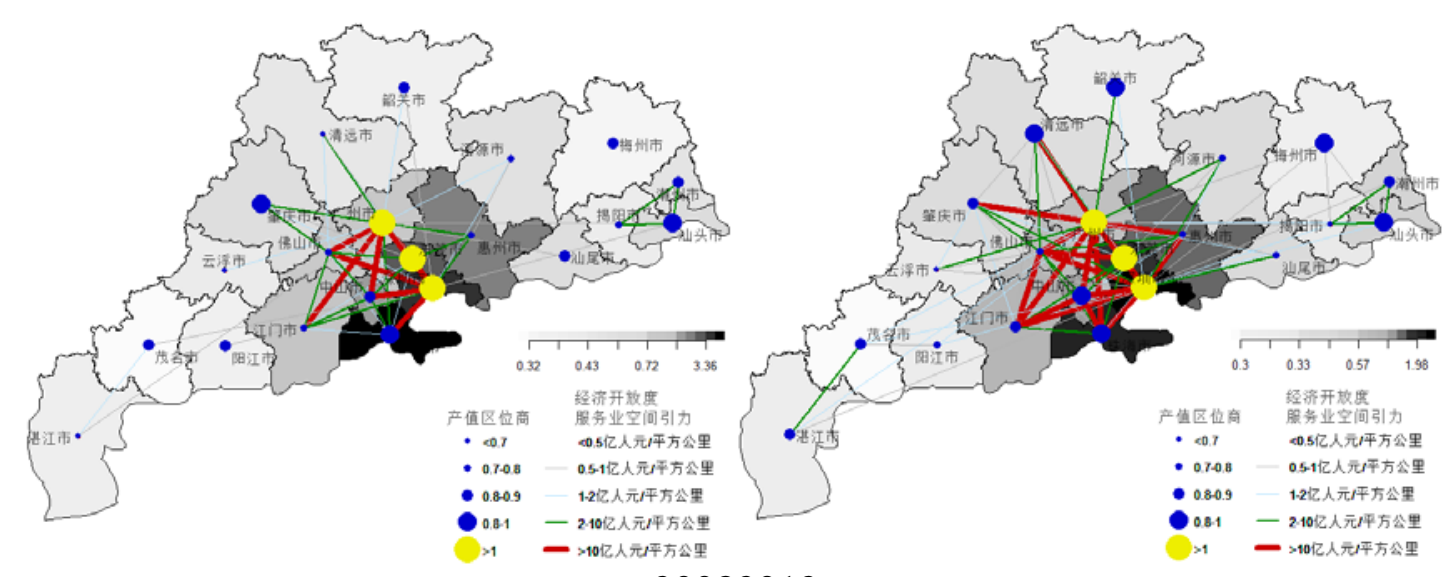

20082012

Fig.2 Spatial fitting between agglomeration, spatial links of Services and distribution of economic openness

Secondly, the number of spatial connections is almost all areas of high economic open areas, that is, service space and economic openness, there are significant spatial fitting relationship. Specifically, to 100 million yuan / square kilometers as the boundary, on the basis of the service industry to explore the basic trends in the development of space links: in 2001, the number of space connections in Guangzhou, Shenzhen, Foshan, Zhuhai, Zhongshan, Jiangmen;in 2005, the number of connections between Dongguan and Zhaoqing also increased significantly, joining the ranks of advanced areas;2008 basically maintain the pattern of 2005 at the same time, Huizhou, Shantou, the number of connections are constantly growing, so the Pearl River Delta all cities are in a higher degree of spatial contact areas;To 2012, Qingyuan, Heyuan, Shanwei and other regions of the number of connections also gradually increased.It can be seen that the spatial openness of the service area is closely related and the degree of interconnection is higher than that of the weakly connected areas. This is also consistent with the actual situation, in general, high economic open areas, Guangdong Province, manufacturing and services developed areas, the higher the degree of openness between the population, information, capital and other resource elements in the region between the higher mobility,as a result, the service industry in the region is becoming closer, and the improvement of the service industry and the social service industry (especially the social service industry) in the service industry is beneficial to attract foreign capital accumulation to a certain extent, accelerate the development of service industry and the evolution of spatial distribution.

In summary, there is a significant spatial fitting relationship between economic openness and spatial connection of service space and agglomeration level. The higher the degree of economic openness, the overall level of manufacturing is generally higher, the manufacturing industry to attract population, capital and other factors together, and then promote the service industry agglomeration, to promote regional industrial restructuring and transformation and upgrading, regional services between the spatial links will gradually increase. Similarly, the increase in service agglomeration and spatial linkages will in turn enhance the investment value of the region, attract more capital and other elements of resources, and indirectly enhance the economic openness of the region. The interaction between the two will be gradually linked to the independent development of the region, and the evolution of the formation of urban agglomeration, the group structure from the initial single-core development to the multi-core circle structure development, and eventually evolved into a spatial network structure, the overall level of economic development Get a big increase.

\section{The conclusions of the study}

Based on the development data of four service industries in Guangdong Province, this paper introduces the spatial gravitational model, constructs the mechanics index of service industry development and discusses the basic characteristics and evolution of spatial relations of service industry in Guangdong Province The paper analyzes the spatial distribution of service industry 
agglomeration, service space connection and regional economic openness, and draws the following conclusions:

(1) Guangdong Province, the development of space-based service industry development situation is obvious, clustering characteristics from the original to Guangzhou as the only gathering core of the "single center" gathering, and gradually evolved into Guangzhou, Shenzhen, Dongguan as the main gathering core Center "agglomeration situation, the regional service industry with the degree of specialization from the gathering center to the surrounding and reduce the spread.

(2) The development potential of the service industry in the prefecture-level cities in Guangdong Province mainly comes from the agglomeration capability of the region itself, and the regional radiation capacity of the developed areas has not been able to play a considerable contribution to the agglomeration ability. The development of service industry in Guangdong Province is still in the main gathering and radiation. The influence of agglomeration center on the surrounding areas is changing from agglomeration to radiation, which indicates that the radiation acceptance capacity of Guangdong Province will gradually play and gather Ability rather than the ability to reverse the role of aggregation.

(3) The spatial relationship between the service industry in Guangdong Province and the development potential of regional service industry and the potential development acceleration have a significant matching relationship in the spatial distribution, and the interaction of spatial gravity between regions is the evolution of spatial structure of regional service industry Which is the core of the spatial structure of the regional service industry. The deepening of the spatial connection of the regional service industry has also promoted the evolution of the spatial structure of the regional service industry, and gradually realized the "core - Edge "structure - circle structure - the transformation of spatial network structure.

(4) There is a significant spatial fitting relationship between the level of service industry agglomeration, the spatial connection of service industry and the opening degree of regional economy, which shows that economic openness, is an important factor to enhance the gathering level of service industry and enhance the spatial connection of service industry. The agglomeration of service industry agglomeration and the deepening of the spatial connection of service industry have accelerated the circulation of various resource elements in the region. By strengthening the economic and social linkages between independent development areas, the population resources have been gathered and developed into urban agglomeration ecosystems. Enhance the investment environment of the whole region, and indirectly enhance the regional economic openness.

\section{References}

[1] Shen Yuming, Qiu Ling, Ren C, et al. Effect of spatial difference of service industry Chinese features [J]. Geographical research, factors and spatial points 2008, 26 (6): 1255-1264.

[2] Xuan Ye. Spatial Agglomeration of Production Service Industry and Improvement of Manufacturing Efficiency - An Empirical Study Based on Spatial Spillover Effect[J]. Spatial spillover effect of Finance \& Economics, 2012 (4): 121-128.

[3] Qiu Ling, Fang Chuanglin. Research on Spatial Agglomeration and Urban Development of Producer Services[J]. City economic geography, 2012, 32 (11): 76-80.

[4] Liu Ming. Heterogeneity of Spatial Distribution in China’s Manufacturing Industry - Analysis Based on GWR and Quantile Regression[J]. Economic issues, 2016 (4): 119-125.

[5] Han Feng, Tian Jialin. Spatial evolution path of producer services agglomeration and space reconstruction of [J]. Technology and innovation management, based on the 2015, 36 (5): 486-491.

[6] Xi, Qiang Min, Chen Xi, Li Guoping. Market potential and spatial distribution of producer services in China. An empirical study based on panel tool model [J]. Geographical science, 2016, 36 (1): $1-9$.

[7] Li Hongjin, Li Sheng. Study on the economic spatial linkages of Urban Agglomeration Based on 
gravity model. An Empirical Study on the urban agglomeration of Pearl River Delta [J]. Journal of South China University of Technology: Social Science Edition, 2011, 13 (1): 19-24.

[8] GuZhaolin, Pang Feng Feng. Spatial linkage and regional division of Chinese urban system based on gravity model [J]. Geography research, 2008, 27 (1): 1-12.

[9] Yang Zhimin, Cheng Xiang Yu, Shao Yuanhai, et al. Study on the spatial linkages of FDI [J]., Zhejiang Province, economic geography, 2013, 33 (011): 21-27.

[10] Lin Xiao, Han Zenglin, Zhao Lin, et al. The spatial pattern and change of financial linkages in China's provinces[J]. Economic geography, 2013, 33 (9): 87-92.

[11] Yang Zhimin, Cheng Xiang Yu, Ye Yafen, et al. Financial spatial relation and grade identification of K-means cluster center [J]. Geography science, 2015, 35 (2): 144-150.

[12] Wang Haijiang, ChanghongLefeng Miao, Ru, et al. China's provincial economic ties the spatial pattern and its change of [J]. Economic geography, 2012, 32 (7): 18-23.

[13] LAN Yisheng. External openness and regional economic growth empirical analysis[J]. Statistical Research, 2002 (2): 19-22.

[14] Luo Jun. Empirical analysis of the influence of economic opening and technological progress on employment of our country's labor force[J]. Financial science, 2014 (4): 61-71. 